\title{
INTERFEROMETRIC OBSERVATIONS FOR O-CONTAINING ORGANIC MOLECULES TOWARDS ORION-KL
}

\author{
Y. C. MINH \\ Daeduk Radio Astronomy Observatory \\ Korea Astronomy Observatory, Hwaam, Yusong, Daejon 305-348, Korea \\ M. OHISHI, D. G. ROH, AND M. ISHIGURO \\ Nobeyama Radio Observatory \\ Nobeyama, Minamimaki, Minamisaku, Nagano 384-13, Japan
}

\begin{abstract}
High spatial resolution observation ( 5 arcsec) were made for $\mathrm{CH}_{3} \mathrm{OH}, \mathrm{HCOOCH}_{3}$, and $\left(\mathrm{CH}_{3}\right)_{2} \mathrm{O}$ toward Orion- $\mathrm{KL}$ using the Nobeyama Millimeter Array. The spatial distribution of $\mathrm{CH}_{3} \mathrm{OH}$ appears to be well elongated along the line connecting IRc2 and "the southern condensation (SC)". The $\mathrm{HCOOCH} 3$ and $\left(\mathrm{CH}_{3}\right)_{2} \mathrm{O}$ emissions appear to be well concentrated to $\mathrm{SC}$ with an angular size of $\sim 6.5$ arcsec. We derive the total column densities $6.8 \times 10^{17} \mathrm{~cm}^{-2}, 1.4 \times 10^{16} \mathrm{~cm}^{-2}$ and $2.7 \times 10^{16} \mathrm{~cm}^{-2}$ for $\mathrm{CH}_{3} \mathrm{OH}, \mathrm{HCOOCH}{ }_{3}$, and $\left(\mathrm{CH}_{3}\right)_{2} \mathrm{O}$, respectively, at the core of SC.
\end{abstract}

\section{INTRODUCTION}

The southern condensation ( $\mathrm{SC}$ ) (or the compact ridge) of Orion-KL region shows an unusually large abundances of oxygen-containing organic molecules, but its chemistry has been poorly understood. Sevaral ideas to explain the large abundances have been proposed : water injection (Blake et al. 1987), methanol injection (Millar, Herbst \& Charnley 1991) to the SC.

In this paper, we made an interferometric observations for the $3 \mathrm{~mm}$ transitions of methanol $\left(\mathrm{CH}_{3} \mathrm{OH}\right)$, methyl formate $\left(\mathrm{HCOOCH}_{3}\right)$, and dimethyl ether $\left(\left(\mathrm{CH}_{3}\right)_{2} \mathrm{O}\right)$ using the Nobeyama Millimeter Array to examine the above ideas.

\section{RESULTS AND DISCUSSION}

Observations were carried out in $1991 \mathrm{March}$ and April. The transitions observed were $\mathrm{CH}_{3} \mathrm{OH} 15_{3}-14_{4} \mathrm{~A}^{-}(88904.09 \mathrm{MHz}), \mathrm{HCOOCH}_{3} 7_{1,6}-6_{1,5} \mathrm{~A}$ and $\mathrm{E}$ (88851.51 and 88843.24 $\mathrm{MHz}$, respectively), and $\left(\mathrm{CH}_{3}\right)_{2} \mathrm{O} 15_{2,13}-15_{1,14} \mathrm{AA}$, $\mathrm{EE}$ and EA+EA (88709.07, 88707.64 and $88706.38 \mathrm{MHz}$, respectively). The synthesized beam provided $5 " .2 \times 4 " .2(\mathrm{FWHM})$ with the position angle of $23^{\circ}$. The CLEANed maps are shown in Fig. 1a-f for the continuum and the integrated intensities of the observed transitions.

Methanol map (Fig. 1b) shows an ellipsoidal shape along IRc2 and SC, and peaks at SC. The distribution is different from those of the previous interfer- 
ometric observations for methanol (Matsakis et al. 1980; Plambeck \& Wright 1988; Wilson et al. 1989). It is essential to observe the several thermal transitions of different energy levels with similar spatial resolutions to understand the excitation and the chemistry of methanol in this region.

The interferometric maps for $\mathrm{HCOOCH}_{3}$ and $\left(\mathrm{CH}_{3}\right)_{2} \mathrm{O}$ were made for the first time. These molecules shows two well concentrated clumps at SC and a.t the west of IRc2 as shown in Fig. 1c. The continuum and the CS emission also show a 'distinct' clump at this position (Murata 1992). These observations indicate that there exists an another condensation with a large gas column density at the west of IRc2. The angular sizes are $\sim 6.5 \operatorname{arcsec}$ and $\sim 4.5 \operatorname{arcsec}($ a.t $2 \sigma \mathrm{rms}$ ) for SC and WC, respectively. The chemistry of WC may be similar to that of $\mathrm{SC}$.

At the core of SC where the emissions of the observed species peak, we derive the total column densities $6.8 \times 10^{17} \mathrm{~cm}^{-2}, 1.4 \times 10^{16} \mathrm{~cm}^{-2}, 2.7 \times 10^{16} \mathrm{~cm}^{-2}$ for $\mathrm{CH}_{3} \mathrm{OH}, \mathrm{HCOOCH}_{3}$, and $\left(\mathrm{CH}_{3}\right)_{2} \mathrm{O}$, respectively, by assuming optically thin emission and an LTE. The rotation temperature $\left(\mathrm{T}_{\text {rot }}\right)$ values used were adopted from Blake et al. (1987), given the uncertainty of $\pm 20 \%$, arbitrary. These values are larger by a factor of 10 - 40 compared with the single dish results (Ohishi 1984; Blake et al. 1987).

Several interesting questions have been raised by our observations: for example, (1) whether the observed abundances for methanol, methyl formate, and dimethyl ether of SC can be explained with the methanol injection model of Millar, Herbst \& Charnley (1991), (2) if so, whether we can explain the source of methanol, (3) whether the morphology of the observed methanol emission indicates any relation with the outflow from IRc2, and so on. Further observations by other transitions are necessary to solve the above questions.

\section{REFERENCES}

Blake, G.A. et al. 1987, $A p J, 315,357$

Matsakis, D.N. et al. 1980, $A p J, \mathbf{2 3 6}, 481$

Millar, T.J., Herbst, E. \& Charnley, S.B. 1991, $A p J, 369,147$

Murata, Y. 1992, Ph.D. Thesis, University of Tokyo

Ohishi, M. 1984, Ph.D. Thesis, University of Tokyo

Plambeck, P.L. \& Wright, M.C.H. 1988, ApJ, 330, L61

Wilson, T.L. et al. 1989, $A \mathscr{E} A, 214,321$ 


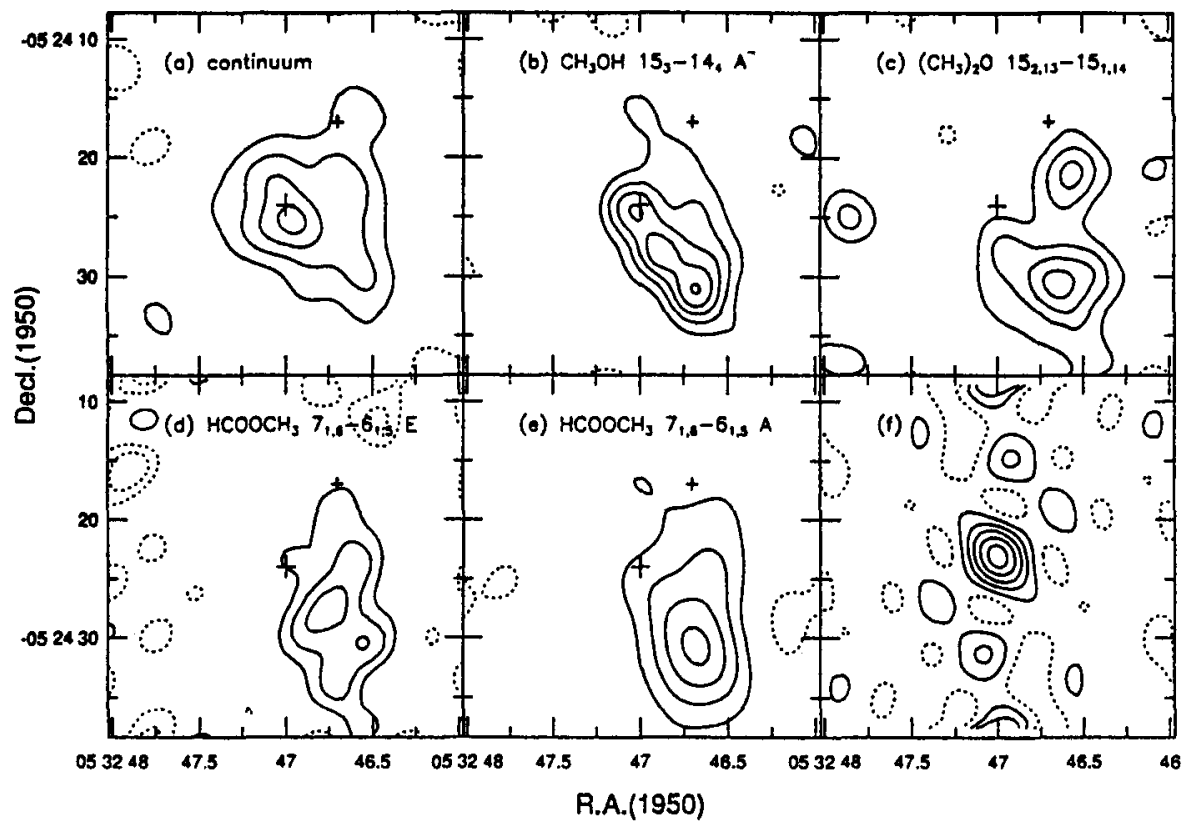

Fig. 1a - 1. Integrated flux intensity maps. Solid lines increase from $1 \sigma$ rms values by $1 \sigma$ and dotted lines decrease from $-1 \sigma$ rms by $1 \sigma$. The larger cross indicates the position of IRc2 and the smaller the BN object. 